\title{
Habilidades de la inteligencia emocional en relación con el consumo de drogas ilícitas en adultos: una revisión sistemática*
}

Emotional intelligence skills in relation to illicit drug use in adults: a systematic review

Habilidades da inteligência emocional em relação ao consumo de drogas ilícitas em adultos: uma revisão sistemática

Julia Lizeth Villarreal Mata

Universidad Autónoma de Nuevo León, México

ORCID: https://orcid.org/0000-0002-8829-0537

Edna Idalia Paulina Navarro Oliva ${ }^{\text {a }}$

Universidad Autónoma de Coabuila, México

enavarro@uadec.edu.mx

ORCID: https://orcid.org/0000-0002-2695-8220

Gerardo Enrique Muñoz Maldonado

Universidad Autónoma de Nuevo León, México

ORCID: https://orcid.org/0000-0002-8937-7909

Linda Azucena Rodriguez Puente

Universidad Autónoma de Coabuila, México

ORCID: https://orcid.org/0000-0003-4040-6171

Luis Arturo Pacheco Pérez

Universidad Autónoma de Sonora, México

ORCID: https://orcid.org/0000-0002-3765-5559
DOI: https://doi.org/10.11144/Javeriana.ie22.hier

Recibido: 03 Abril 2019

Aprobado: 12 Diciembre 2019

Publicado: 22 Julio 2020

\section{Resumen:}

Introducción: La evaluación de la inteligencia emocional (IE) es centro de interés para profesionales de diversos campos de la salud y la educación. Objetivo: Identificar el estado del arte de las habilidades de la IE y las posibles relaciones con el consumo de drogas en adultos. Metodología: Se utilizó como guía la Preferred Reporting Items for Systematic Reviews and Meta-Analyses (PRISMA). Se formuló la pregunta de investigación para dar respuesta al objetivo, se definieron los criterios de selección y, posteriormente, se buscaron artículos para el periodo 2007-2018 en las bases de datos PubMed, Scopus, EBSCO, Web of Science y OVID. Mediante el programa EndNote, se evaluaron los estudios para el análisis de los datos y sintetizarlos. Resultados: Se identificaron 1172 artículos a través de las bases de datos y se eligieron 15 estudios, de los cuales solo 3 cumplieron con los criterios de elegibilidad y calidad metodológica. Una baja IE da por resultado un consumo más intenso y frecuente de sustancias ilegales; por ende, una mayor dificultad en el manejo y regulación emocional. Conclusiones: La investigación de la IE y las adicciones son relativamente nuevas y se necesitan más estudios para comprender esta relación con mayor detalle.

Palabras clave: inteligencia emocional, emociones, drogas ilícitas.

\section{Abstract:}

Introduction: The evaluation of emotional intelligence (EI) is of particular interest for professionals in various fields of health and education. Objective: To identify the state of the art of research on EI skills and the possible relationships with drug use in adults.

Notas de autor

\footnotetext{
a Autora de correspondencia. Correo electrónico: enavarro@uadec.edu.mx
} 
Methodology: The Preferred Reporting Items for Systematic Reviews and Meta-Analyzes (PRISMA) was used as a guide. First, the research question was defined to provide an answer for the objective; then, the selection criteria were established; and, finally, articles for the period 2007-2018 were found through a search in the Pub Med, Scopus, EBSCO, Web of Science, and OVID databases. Studies were then assessed and analyzed using EndNote and subsequently summarized to extract their corresponding theses. Results: A total 1,172 articles were identified across databases, out of which 15 studies were chosen and from these only 3 met the eligibility criteria and methodological quality. The sample shows that a lower EI leads to a more intense and frequent consumption of illegal substances, thus leading to greater difficulties in emotional management and regulation. Conclusions: Research on EI and addictions is relatively new, and more studies are needed to understand this relationship in greater detail.

Keywords: emotional intelligence, emotions, illicit drugs.

\section{Resumo:}

Introdução: a avaliação da inteligência emocional (IE) é centro de interesse para profissionais de diversos campos da saúde e do ensino. Objetivo: Identificar o estado da arte das habilidades da IE, inteligência emocional, e os possíveis relacionamentos com consumo de drogas em adultos. Metodologia: A Preferred Reporting Items for Systematic Reviews and Meta-Analyses (PRISMA) foi usada como guia. Primeiro, a pergunta de pesquisa para dar resposta ao objetivo foi formulada, definiram-se os critérios de seleção e, posteriormente, a busca de artigos no período 2007-2018 foi feita. As bases de dados foram Pub Med, Scopus, EBSCO, Web of Science e OVID. Por meio do programa EndNote avaliaram-se os estudos para realizar a análise, síntese e descrição dos dados. Resultados: Foram identificados 1,172 artigos das bases de dados e elegeram-se 15 estudos, dos quais apenas três preencheram os critérios de elegibilidade e qualidade metodológica. Uma amostra baixa de IE resulta em um consumo mais intenso e frequente de sustâncias ilegais, portanto, maior dificuldade no maneio e regulação emocional. Conclusões: A pesquisa da IE e as adições é relativamente nova e são precisos mais estudos para compreender essa relação com maior detalhe.

Palavras-chave: inteligência emocional, emoções, drogas ilegais.

\section{Introducción}

El consumo de drogas ilícitas se ha extendido globalmente y es considerado un complejo problema de salud pública $(1,2)$. Esta problemática origina múltiples consecuencias negativas en las esferas física, psicológica y social. En el mundo, se calcula que más de 250 millones de personas han consumido drogas alguna vez en la vida, de las cuales aproximadamente 29,5 millones padecen trastornos o complicaciones provocadas por el consumo de alguna sustancia ilícita (2).

En México, el consumo de drogas ilícitas muestra una tendencia al aumento. De acuerdo con lo estipulado en la Encuesta Nacional de Consumo de Drogas, Alcohol y Tabaco (3), se menciona que el 0,3\% de la población ha consumido cualquier droga alguna vez en la vida, el 2,9\% en el último año y el 1,5\% en el último mes, de los cuales el 0,6\% ya presenta una posible dependencia al consumo de drogas en el último año. Este panorama resulta alarmante y por ello el sector de la salud promueve un enfoque integral para prevenir y disminuir el número de usuarios, mitigar los daños a la salud que causa el abuso y proveer información y tratamiento a los consumidores adictos, con el objetivo de rehabilitarlos y reintegrarlos a la sociedad (4).

En este sentido, las medidas de prevención y tratamiento para las adicciones deben estar sustentadas en información científica, confiable y completa sobre la naturaleza, la magnitud y las características del fenómeno, ya que la adicción a las drogas depende de una amplia variedad de factores que van desde los genéticos hasta variables de personalidad o la situación sociocultural $(5,6,7)$. Además, ciertos autores mencionan que las personas que presentan problemas de adicción se caracterizan por presentar dificultades para el manejo de las emociones 8,9,10), y pueden recurrir al consumo de drogas como forma externa de autorregulación y así mitigar estados emocionales negativos o con la finalidad de desarrollar estados emocionales más placenteros $(11,12)$. Cabe mencionar que dentro de los diversos factores que llevan a una persona a consumir drogas, desempeña un papel muy importante la falta de gestión para regular las emociones.

Las personas con competencias o habilidades para manejar emociones tienen un alto grado de inteligencia emocional (IE); se caracterizan por presentar menos emociones negativas, por ser más capaces de llegar al autocontrol y por contar con una buena regulación emocional, lo cual les facilita la toma decisiones. De 
acuerdo con lo anterior, el estudio de las habilidades relacionadas con la IE como factores influyentes para el desarrollo de adicciones ha sido tema de interés para las nuevas investigaciones (12).

Se entiende como IE el conjunto de habilidades, referentes al ámbito emocional y social, que influye en el afrontamiento de las demandas de nuestro medio. Dicha habilidad se basa en la capacidad del individuo de ser consciente, comprender, controlar, regular y expresar sus emociones de manera efectiva (13). El modelo de Bar-On se fundamenta en las habilidades y considera la IE un conjunto de elementos emocionales, personales y sociales que influyen en la habilidad general para adaptarse de manera activa a las presiones y demandas del ambiente. Se compone de cinco elementos: 1) el intrapersonal, referente a la comprensión de si\# mismo, asertividad, autoconcepto, autorrealización e independencia, y se entiende como la habilidad de ser consciente, comprender y relacionarse. 2) El interpersonal, que incluye empatía, relaciones interpersonales y responsabilidad social, e implica la habilidad para regular emociones e impulsos fuertes. 3) La adaptabilidad, que hace referencia a la solución de problemas, es decir, la habilidad de identificar los problemas y generar y efectuar soluciones efectivas. 4) El estado de ánimo, que es la habilidad para ajustarse a los cambios y resolver problemas personales y sociales. Y 5) el componente del manejo de estrés, que involucra la habilidad de tener una visión positiva y optimista (13).

Investigaciones recientes hacen hincapié en la importancia que tienen las emociones en el consumo de sustancias y señalan que la habilidad para descodificar y diferenciar emociones o la regulación emocional son factores clave en las adicciones (14); además, abordan que el factor emocional es una habilidad que se encuentra dañada en la población que consume drogas (15). En este sentido, se menciona que probablemente los individuos con dificultades para regular sus estados emocionales están más predispuestos al consumo temprano de sustancias.

Se han encontrado correlaciones entre la IE y el abuso de sustancias $(10,17,18)$; así como que niveles bajos IE están asociados con un consumo mayor y más problemático de tabaco, alcohol y drogas (6,12-15,18-24). Salovey y Mayer (25) definieron la IE como "la capacidad para supervisar los sentimientos y las emociones de uno mismo y de los demás, de discriminar entre ellos y usar esta información para la orientación de la acción y el pensamiento propio". Actualmente, existen publicaciones que evidencian que diferentes disciplinas a lo largo del tiempo (26) han abordado el constructo de la IE: la educación $(27,28)$, la salud (29,30), la empresa (31-34) y el campo científico en general (35-38).

Sin embargo, en la disciplina de enfermería, la investigación de la IE es un constructo abordado recientemente, por la poca evidencia en investigación de la IE aplicada al cuidado realizado por la profesión de enfermería, enfocado en la prevención y tratamiento del consumo de drogas (39), ya que al fungir como un factor protector de conductas de riesgo, puede ser utilizada como una medida preventiva en adicciones $(40,41,42,43,44,45,46)$.

Por lo expuesto, el objetivo del artículo fue identificar el estado del arte de las habilidades de la IE y las posibles relaciones con el consumo de drogas en adultos mediante una revisión sistemática utilizando el método ThePreferred Reporting Items for Systematic Reviews and Meta-Analyses (PRISMA). Como objetivos específicos se pretende profundizar en el conocimiento de las características y habilidades de la IE y su relación con las drogas. Se espera que los resultados aporten conocimiento y sirvan de base para el desarrollo de intervenciones que incluyan la IE en la prevención y el tratamiento en las adicciones.

\section{Método}

Para llevar a cabo la revisión de la literatura sobre el tema se tomaron en cuenta los pasos propuestos por el Manual Cochrane (47). Primero se formuló la pregunta de investigación: ¿cuál es el estado del arte de las habilidades de la IE y las posibles relaciones con el consumo de drogas en adultos? Luego se definieron los 
criterios de selección. Posteriormente, se buscaron y seleccionaron los artículos pertinentes. Enseguida, se evaluaron los estudios para analizar los datos. Finalmente, se sintetizaron.

\section{Estrategia de búsqueda}

Los artículos para revisión se identificaron a partir de las búsquedas en las bases de datos de literatura académica (PubMed, Scopus, Ebsco, Web of Science y OVID), en el periodo del 2007 al 2018. Para la identificación de los artículos se utilizó una combinación de los descriptores DeCS y MeSH en conjunto con los operadores booleanos AND, OR y NOT. Dicha estrategia fue probada primeramente en PubMed y después en las otras bases de datos consultadas. La estrategia incluyó los términos adultos y adultos jóvenes combinados con las palabras inteligencia emocional, abuso de drogas, abuso de sustancias, drogas ilícitas.

\section{Criterios de inclusión y exclusión}

Se incluyeron todos los artículos que cumplieron con los siguientes criterios: 1) el artículo es de investigación original publicado en una revista revisada por pares; 2) estudios publicados en español e inglés; 3) en cuanto a metodología, se incluyeron los estudios de casos y controles; 4) los participantes del estudio son humanos; 5) el artículo reporta el uso de cualquier droga ilegal, y 6) los participantes del estudio son adultos ( 19 a 90 años) y adultos jóvenes (19 a 24 años). Se excluyeron los estudios cualitativos, las revisiones sistemáticas, las pautas basadas en la evidencia, revisiones de literatura, así como los comentarios y artículos de opinión experta.

En total se obtuvieron 1172 artículos de todas las bases de datos revisadas; posteriormente, se procedió a la lectura de títulos y resúmenes como un primer filtro para los artículos que fueron seleccionados para el análisis crítico, con la finalidad de realizar la búsqueda de las principales variables y la relación entre la IE y el consumo de drogas en la población de interés. Se utilizaron herramientas de trabajo (47) como la lectura crítica, subrayado y cuadro de análisis (nivel de evidencia, diseño, tamaño de muestra e instrumentos utilizados). En la figura 1 se muestra el proceso de inclusión y exclusión de los artículos en esta revisión sistemática. 


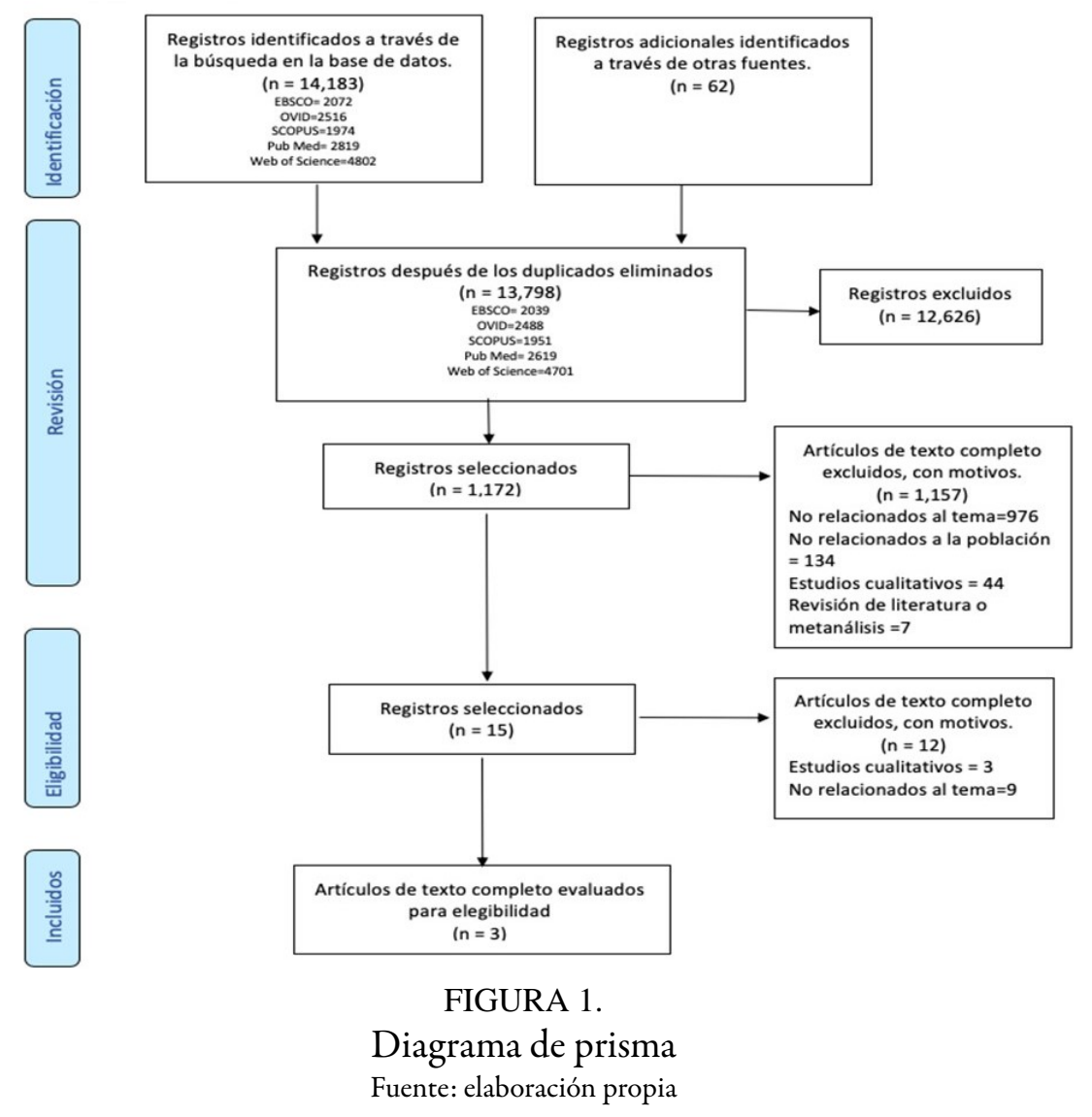

\section{Resultados}

De acuerdo con los estudios analizados, se identificó la relación entre las habilidades de la IE y el consumo de drogas ilícitas. En la presente revisión se identificaron 3 estudios hechos en el periodo 2007-2018 (figura 1), en cuanto a las características de la muestra. Todos fueron adultos mayores de 18 años con trastorno por abuso de sustancias, a excepción de los grupos control. Solo en el $66,6 \%$ se estudia la IE de forma específica $(48,49)$, y el restante utiliza conceptos como "control de emociones", "manejo de emociones" o "regulación de emociones" (50). Parolin et al. (49) y Craig et al. (50) evaluaron y describieron la identificación de emociones, así como la respuesta de las emociones en usuarios dependientes y no dependientes de drogas. Entre tanto, Fox et al. (51) examinaron la dificultad en la regulación de las emociones y control de impulsos asociada con la abstinencia en el trastorno por uso de sustancias.

En referencia a la clasificación anterior, en los dos artículos restantes, Parolin et al. (49) abordaron la IE desde un enfoque de funcionamiento y sensibilidad; mientras que Craig et al. (50) analizaron la relación entre el uso del éxtasis, la IE, el estado de ánimo y los estilos de crianza. Los análisis estadísticos utilizados fueron pruebas t de Student, análisis de varianza, análisis de covarianza, correlaciones de Pearson, y chi cuadrado $(\chi$.$) , así como análisis de mediación. Los resultados de los análisis y los instrumentos empleados se muestran$ en la tabla 1. 
TABLA 1.

Resultados de la revisión sistemática, publicados en el periodo del 2007 al 2018

\begin{tabular}{|c|c|c|c|c|c|}
\hline Estudio & Diseño & $\begin{array}{l}\text { Tamańo de } \\
\text { muestra }\end{array}$ & Características de la muestra & Evaluación de la IE & Resultados \\
\hline \multirow[t]{2}{*}{$\begin{array}{l}\text { Fox et } \\
\text { al. }(51)\end{array}$} & \multirow[t]{2}{*}{$\begin{array}{l}\text { Casos y } \\
\text { controles }\end{array}$} & \multirow[t]{2}{*}{110 personas } & $\begin{array}{l}60 \text { personas dependientes de la } \\
\text { cocaína ( } 28 \text { mujeres y } 32 \\
\text { hombres) }\end{array}$ & \multirow[t]{2}{*}{$\begin{array}{l}\text { Escala de Dificultad en la } \\
\text { Regulación de Emociones } \\
\text { (DERS) }\end{array}$} & $\begin{array}{l}\text { En comparación con los controles, } \\
\text { los individuos dependientes de la } \\
\text { cocaína informaron las dificultades } \\
\text { relacionadas con las emociones, la } \\
\text { gestión de las emociones y el } \\
\text { control de los impulsos en la } \\
\text { primera semana de abstinencia. }\end{array}$ \\
\hline & & & $\begin{array}{l}50 \text { voluntarios sanos }(24 \\
\text { mujeres y } 26 \text { hombres })\end{array}$ & & $\begin{array}{l}\text { En la puntuación DERS total } \\
(85,8 \pm 22,5 \text { en comparación con } \\
60,9 \pm 15,0 ; p<0,01)\end{array}$ \\
\hline \multirow{4}{*}{$\begin{array}{l}\text { Parolin } \\
\text { et al. } \\
\text { (49) }\end{array}$} & \multirow[t]{4}{*}{$\begin{array}{l}\text { Casos y } \\
\text { controles }\end{array}$} & \multirow{4}{*}{$\begin{array}{l}68 \text { sujetos de } \\
18 \text { a } 24 \text { años } \\
\text { de edad }\end{array}$} & $(\mathrm{n}=41)$ adultos consumidores & $\begin{array}{l}\text { Escala de Alexitimia de } \\
\text { Toronto (TAS-20) }\end{array}$ & \multirow{4}{*}{$\begin{array}{l}\text { El grupo con adicciones en } \\
\text { comparación con el grupo control } \\
\text { mostró dificultad significativa para } \\
\text { identificar emociones }\left(\chi^{2}=5,460 ;\right. \\
p=0,025) \text { y se clasificaron como } \\
\text { alexitímico en la puntuación total } \\
\text { TAS-20 }\left(\chi^{2}=8,361 ; p=0,005\right)\end{array}$} \\
\hline & & & $\begin{array}{l}(\mathrm{n}=27) \text { sin consumo de } \\
\text { sustancias }\end{array}$ & $\begin{array}{l}\text { La Escala de Alexitimia del } \\
\text { Observador (OEA) }\end{array}$ & \\
\hline & & & $\begin{array}{l}\text { (no se reporta que tipo de } \\
\text { droga) }\end{array}$ & $\begin{array}{l}\text { Inventario de Inteligencia } \\
\text { Emocional Bar-On (EQ-i) }\end{array}$ & \\
\hline & & & & $\begin{array}{l}\text { Escala de Búsqueda de } \\
\text { Sensaciones - forma VI } \\
\text { (SSS-VI). }\end{array}$ & \\
\hline
\end{tabular}

Fuente: elaboración propia

TABLA 1 (cont.)

Resultados de la revisión sistemática, publicados en el periodo del 2007 al 2018

\begin{tabular}{|c|c|c|c|c|c|}
\hline Estudio & Diseńo & $\begin{array}{l}\text { Tamańo de } \\
\text { muestra }\end{array}$ & Características de la muestra & Evaluación de la IE & Resultados \\
\hline \multirow[t]{5}{*}{$\begin{array}{l}\text { Craig et } \\
\text { al. }(50)\end{array}$} & \multirow[t]{5}{*}{$\begin{array}{l}\text { Casosy } \\
\text { controles }\end{array}$} & \multirow[t]{5}{*}{150 personas } & \multirow{5}{*}{$\begin{array}{l}34 \text { (no consumían sustancias } \\
\text { ilícitas) } 38 \text { (consumían solo } \\
\text { cannabis) y } 78 \text { (consumían } \\
\text { múltiples drogas, 3,4- } \\
\text { metilendioximetanfetamina } \\
\text { (MDMA; "éxtasis", } \\
\text { anfetaminas, cocaína) }\end{array}$} & $\begin{array}{l}\text { Cuestionario de estilo de } \\
\text { crianza }\end{array}$ & \multirow{5}{*}{$\begin{array}{l}\text { No hubo diferencias } \\
\text { significativas entre los grupos en } \\
\text { cuanto a la } \operatorname{IE~} F(2,147)=0,020 ; \\
p>0,05 \text {. }\end{array}$} \\
\hline & & & & $\begin{array}{l}\text { Cuestionario de IE } \\
\text { desarrollado por Schutte, } \\
\text { et al. (1998) }\end{array}$ & \\
\hline & & & & $\begin{array}{l}\text { Matrices Progresivas de } \\
\text { Raven }\end{array}$ & \\
\hline & & & & $\begin{array}{l}\text { Prueba Nacional de } \\
\text { Lectura en Adultos } \\
\text { (NART) }\end{array}$ & \\
\hline & & & & $\begin{array}{l}\text { Lista de verificación de } \\
\text { adjetivos del estado de } \\
\text { ánimo. }\end{array}$ & \\
\hline
\end{tabular}

Fuente: elaboración propia

En los datos sociodemográficos Fox et al. (51) reportan que los pacientes adictos a la cocaína y los participantes del grupo control difirieron significativamente por edad $(\bar{x}=37,2 ; \mathrm{DE} \pm 5,9$ y $\bar{x}=33,6 ; \mathrm{DE} \pm 10,0$; $p=0,02)$. Esto es diferente a lo reportado por Parolin et al. (49), que reportan que el $54 \%$ de la muestra eran varones y la edad media era de 21 años $(\mathrm{DE} \pm 2,1)$ y es similar a lo reportado por Craig et al. (50), para quienes la media de edad fue de $\bar{x}=21,23(\mathrm{DE}=1,95)$.

En cuanto a la IE, Fox et al. (51) reportaron que los pacientes usuarios de cocaína mostraron puntuaciones más altas al inicio que los controles en la puntuación Escala de Dificultad en la Regulación de Emociones (DERS) total $(\bar{x}=85,8 ; \mathrm{DE} \pm 22,5)$ en comparación con el grupo control $(\bar{x}=60,9 ; \mathrm{DE} \pm 15,0 ; p<0,01)$, pero no hubo diferencias al alta $(\bar{x}=77,6 ; \mathrm{DE} \pm 20,7)$. Muy distinto a lo reportado por Parolin et al. (49), para quienes había una diferencia significativa entre los dos grupos, donde los usuarios de drogas mostraron mayor dificultad clínicamente significativa en la identificación de las emociones $(\chi 2=5,460 ; p=0,025)$. Además, en 
cuanto a la IE, el 70,8\% de los jóvenes con adicción mostraron en la habilidad manejo del estrés por debajo de la media, y el 65,9\% en la escala general del estado de ánimo. En síntesis, para todas las subescalas, menos del $30 \%$ de los sujetos no clínicos alcanzó un nivel insuficiente de las capacidades emocionales. Por el contrario, Craig et al. (50) reportan que no hubo diferencias de grupo sustanciales en la IE $(\bar{x}=119,32 ; \mathrm{DE}=12,76)$.

\section{Discusión}

El presente artículo otorga evidencia de que los niveles más bajos de IE corresponden a un consumo más intenso y problemático de sustancias ilegales. Se muestra también que dicho consumo excesivo da por resultado una mayor dificultad en el manejo emocional; similar a lo reportado en otras revisiones sistemáticas (13), por lo que vale mencionar que el estudio de la IE, así como la descodificación, el reconocimiento y el manejo de las emociones, protagoniza un papel clave en el trastorno por uso de sustancias y adicciones.

En cuanto a los factores fisiológicos, no se encontró evidencia sobre los posibles efectos del género, la edad y el origen étnico en la relación entre el uso de sustancias y la IE, similar a lo reportado por Kun y Demetrovics (14). Sin embargo, la investigación acerca de estos factores sería necesaria, ya que sons significativos tanto en el uso de sustancias como en la IE. Por otro lado, en los artículos revisados, en la mayoría de los casos la IE se mide con instrumentos de autoevaluación ampliamente utilizados y probados, corroborando lo encontrado por Sánchez-Teruel y Robles-Bello (52) y Resurrección et al. (53), quienes reportan alta recurrencia a cuestionarios de autoevaluación, probablemente por lo fácil, rápido y económico de la aplicación; no obstante, esto pudiese significar la posibilidad de sesgo y datos erróneos.

Con relación a las habilidades de la IE, hay discrepancias en los tres estudios, ya que uno solo mide el constructo de emociones y reporta que en los usuarios de drogas es menor su manejo (51). El otro estudio indica las mediciones de la IE y menciona que las habilidades están disminuidas en las personas que usan drogas; sin embargo, no reporta el tipo de sustancias (49). El tercer texto, de forma contraria, no indica cambios en el manejo regulación de las habilidades emocionales (50). Lo anterior puede estar influenciado por la cantidad de la muestra, el tipo de sustancia y el tiempo en el que se colectaron los datos.

Ahora bien, algunos de los estudios no abarcan la clasificación del tipo de sustancias, tampoco el tipo de uso (ocasional, recreación o diario), y ningún estudio menciona si las personas comienzan a usar drogas porque no pueden regular las emociones o el uso de drogas provoca problemas en el manejo de las emociones; por lo que es conveniente investigar si la falta de capacidad endógena para la regulación emocional conduce a la búsqueda de factores reguladores exógenos, como las drogas.

\section{Conclusión}

Los estudios revisados muestran que una baja IE da por resultado un consumo más intenso y frecuente de sustancias ilegales; en consecuencia, tienen una mayor dificultad en el manejo y regulación emocional. La investigación de la IE y las adicciones son relativamente nuevas y se requieren más estudios para comprender esta relación en mayor detalle. Entre las limitaciones de este trabajo hay que destacar que algunos de los estudios consultados no se incluyeron en la revisio\#n por no presentar un grupo control o porque las diferentes medidas o variables que se utilizaban presentaban bajos niveles de fiabilidad o validez. Así mismo, la diversidad entre las habilidades de la IE es muy amplia y esto puede generar conflictos en el momento de revisar el enfoque de los términos. 


\section{Referencias}

1. Organización Mundial de la Salud. La dimensión de salud pública del problema mundial de las drogas. Ginebra; 2016.

2. Oficina de las Naciones Unidas contra la Droga y el Delito. Informe mundial sobre las drogas 2017. Santiago; 2017.

3. Instituto Nacional de Psiquiatría Ramón de la Fuente Muñiz; Instituto Nacional de Salud Pública Comisión Nacional Contra las Adicciones Secretaría de Salud. Encuesta Nacional de Consumo de Drogas Alcohol y Tabaco 2016-2017: reporte de drogas [internet]. Ciudad de México: INPRFM; 2017. Disponible en: https://www.in prf.gob.mx

4. Villatoro J, Medina-Mora ME, Fleiz Bautista C, Moreno López M, Oliva Robles N, Bustos Gamiño M, et al. El consumo de drogas en México: resultados de la Encuesta Nacional de Adicciones, 2011. Salud Mental [Internet]. 2012;35(6):447-57. Disponible en: http://www.scielo.org.mx/scielo.php?script=sci_arttext\&pid=S0185-332 52012000600001

5. Gilbert DG. Gender differences in tobacco use and effects. En: Smoking: individual differences, psychopathology and emotion. Washington DC: Taylor \& Francis; 1995. p. 177-91. https://doi.org/10.4324/9781315825205

6. Kassel JD, Stroud LR y Paronis CA. Smoking, stress and negative affect: correlation, causation and context across stages of smoking. Psychol Bull. 2003;129(2):270-304. https://doi.org/10.1037/0033-2909.129.2.171.

7. Terracciano A, Costa PT Jr. Smoking and the five-factor model of personality. Addiction. 2004;99(4):472-81. htt ps://doi:10.1111/j.1360-0443.2004.00687.x

8. Novak SP, Clayton RR. The influence of school environment and self-regulation on transitions between stages of cigarette smoking: a multilevel analysis. Health Psychol. 2001;20:196-207. https://doi.org/10.1037/0278-613 3.20.3.196

9. Ciarrochi J, Forgas JP, Mayer JD, editores. Emotional intelligence and everyday life. New York: Psychology Press; 2001. p. 76-77.

10. Brackett MA, Mayer JD. Convergent, discriminant and incremental validity of competing measures of emotional intelligence. Pers Soc Psychol Bull. 2003;29(9):1147-58. https://doi.org/10.1177/0146167203254596

11. Trinidad DR, Johnson CA. The association between emotional intelligence and early adolescent tobacco and alcohol use. Pers Individ Differ. 2002;32(1):95-105. https://doi.org/10.1016/S0191-8869(01)00008-3

12. Fernández SB, Jorge V, Bejar LE. Función protectora de las habilidades emocionales en la prevención del consumo de tabaco y alcohol: una propuesta de intervención. Psicooncología. 2009;6(1): 243-56. https://dx.doi.org/10 $.5209 /$ PSIC

13. Bar-On R. The Bar-On Emotional Quotient Inventory (EQ-i): rationale, description and summary of psychometric properties. En: Geher G, editor. Measuring emotional intelligence: common ground and controversy. Nova Science Publishers; 2004. p. 115-45.

14. Kun B, Demetrovics Z. Emotional intelligence and addictions: a systematic review. Subs Use Misuse. 2010;45(7-8):1131-60. https://doi:10.3109/10826080903567855

15. Fernández-Serrano MJ, Perales López JC, Moreno-López L, Santos-Ruiz A, Pérez-García M, Verdejo García A. Impulsividad y compulsividad en individuos dependientes de cocaína. Adicciones. 2012;24(2):105-13. https:/ /doi.org/10.20882/adicciones.102

16. Limonero JT, Gómez-Romero MJ, Fernández-Castro J, Tomás-Sábado J. Influencia de la inteligencia emocional percibida y la impulsividad en el abuso de cánnabis en jóvenes. Ansiedad Estrés [Internet]. 2013;19(2-3):223-34.Disponible en: https://www.researchgate.net/publication/257366783_Influencia_de_la_inteligencia_emocional_percibi da_y_la_Impulsividad_en_el_abuso_de_cannabis_en_jovenes_Influence_of_perceived_emotional_intelligen ce_and_impulsivity_in_cannabis_abuse_in_young/citation/download

17. Riley H, Schutte NS. Low emotional intelligence as a predictor of substance-use problems. J Drug Educ. 2003;33(4):391-8. https://doi.org/10.2190/6DH9-YT0M-FT99-2X05 
18. Schutte NS, Malouff JM, Hine DW. The association of ability and trait emotional intelligence with alcohol problems. Addict Res Theory. 2011;19(3):260-5. https://doi.org/10.3109/16066359.2010.512108

19. Canto J, Fernández-Berrocal P, Guerrero F, Extremera N. Función protectora de las habilidades emocionales en las adicciones. En: Romay Martínez J, García Mira R, editores. Psicología social y problemas sociales. Madrid: Biblioteca Nueva; 2005. p. 583-90.

20. García del Castillo JA, García del Castillo-López Á, Gázquez Pertusa M, Marzo Campos JC. La inteligencia emocional como estrategia de prevención de las adicciones. Salud y Drogas [Internet]. 2013;13(2):89-97. Disponible en: https://www.redalyc.org/articulo.oa?id=83929573001

21. Hill EM y Maggi S. Emotional intelligence and smoking: Protective and risk factors among Canadian young adults. Personality and Individual Differences. 2011;51(1):45-50. https://doi.org/10.1016/j.paid.2011.03.008

22. Maddi SR, Erwin LM, Carmody CL, Villarreal BJ, White M, Gundersen KK. Relationship of hardiness, grit, and emotional intelligence to internet addiction, excessive consumer spending, and gambling. J Posit Psychol. 2013;8(2):128-34. https://doi.org/10.1080/17439760.2012.758306

23. Muñoz G, Rodolfo A. Tratamiento ambulatorio del paciente farmacodependiente: propuesta de intervención psicosocial basada en inteligencia emocional [tesis de doctorado]. Universidad de Oriente; 2009.

24. Ruiz-Aranda D, Cabello R, Salguero JM, Castillo R, Extremera N, Fernández-Berrocal P. Los adolescentes malagueños ante las drogas: la influencia de la inteligencia emocional. Málaga: GEU; 2010.

25. Salovey P, Mayer JD. Emotional intelligence. Imagin Cogn Pers. 1990;9(3):185-211. https://doi.org/10.2190/D UGG-P24E-52WK-6CDG

26. Fredrickson BL. What good are positive emotions? Rev Gen Psychol. 1998;(2):300-19. https://doi.org/10.1037 /1089-2680.2.3.300

27. Avilés JM. Bullying: el maltrato entre iguales. Agresor, víctimas y testigos en la escuela. Salamanca: Amarú; 2006.

28. Barchard K. Does emotional intelligence assist in the prediction of academic success? Educ Psychol Meas. 2003;63(5):840-58. https://doi.org/10.1177/0013164403251333

29. Augusto-Landa JM, Berríos-Martos M, López-Zafra E, Aguilar-Luzón MC. Relación entre burnout e inteligencia emocional y su impacto en la salud mental, bienestar y satisfacción laboral en profesionales de enfermería. Ansiedad Estrés. 2006;12:479-93.

30. Bisquerra R. Educación emocional y bienestar. Barcelona: Praxis; 2000.

31. Abraham R. The roll of job controls as a moderator of emotional dissonance and emotional intelligence-outcome relationships. J Psychol. 2000;134:169-84. https://doi.org/10.1080/00223980009600860

32. Caruso D, Salovey P. El directivo emocionalmente inteligente: la inteligencia emocional en la empresa. Málaga: Algaba; 2005.

33. Goleman D. Inteligencia emocional. Barcelona: Kairós; 1996.

34. Goleman D. La práctica de la inteligencia emocional. Barcelona: Kairós, 1999.

35. Bar-On R, Parker JDA. Handbook of emotional intelligence. San Francisco: Jossey; 2000.

36. Bermúdez MP, Teva I, Sánchez A. Análisis de la relación entre inteligencia emocional, estabilidad emocional y bienestar psicológico. Univ Psychol [internet]. 2003;2:27-32. https://www.redalyc.org/pdf/647/64720105.pdf

37. Ciarrochi J, Forgas JP, Mayer JD. Emotional intelligence in everyday life. Philadelphia: Psychology Press/Taylor and Francis; 2006.

38. Mayer JD, Salovey P. The intelligence of emotional intelligence. Intelligence. 1993;17:433-42.

39. Mayer JD, Salovey P. What is emotional intelligence? En: Salovey P, Sluyter D, editores. Emotional development and emotional intelligence: educational implications. New York: Basic Books; 1997.

40. Aradilla Herrero A. Inteligencia emocional y variables relacionadas en enfermería [tesis]. Barcelona: Universidad de Barcelona; 2013. Disponible en: http://hdl.handle.net/10803/119774

41. Clarke $\mathrm{N}$. The impact of a training programme designed to target the emotional intelligence abilities of project managers. Int J Project Manage. 2010;28(5):461-8. https://doi:10.1016/j.ijproman.2009.08.004 
42. Cook GL, Bay D, Visser B, Myburgh JE, Njoroge J. Emotional intelligence: the role of accounting education and work experience. Issues in Accounting Education. 2011;26(2):267-86.https://doi.org/10.2308/iace-10001

43. Di Fabio A, Kenny ME. Promoting emotional intelligence and career decision making among Italian high school students. J Career Assess. 2011;19(1):21-34. https://doi.org/10.1177/1069072710382530

44. Lindebaum D. Rhetoric or remedy? A critique on developing emotional intelligence. Acad Manag Learn Educ. 2009;8(2):225-37.

45. Nelis D, Quoidbach J, Mikolajczak M, Hansenne M. Increasing emotional intelligence: (How) is it possible? Person Indiv Diff. 2009;47(1):36-41. https://doi.org/10.1016/j.paid.2009.01.046

46. Qualter P, Gardner KJ, Pope DJ, Hutchinson JM, Whiteley HE. Ability emotional intelligence, trait emotional intelligence, and academic success in British secondary schools: A 5-year longitudinal study. Learn Indiv Diff. 2012;22(1):83-91. https://doi.org/10.1016/j.lindif.2017.06.005

47. Ruiz-Aranda D, Castillo R, Salguero JM, Cabello R, Fernández-Berrocal P, Balluerka N. Short-and midterm effects of emotional intelligence training on adolescent mental health. J Adolesc Health. 2012;51(5):462-7. https://d oi.org/10.1016/j.jadohealth.2012.02.003

48. Higgins JPT, Green S, editores. Cochrane handbook for systematic reviews of interventions version 5.1.0 [internet]. The Cochrane Collaboration; 2011. Disponible en: http://www.cochrane-handbook.org.

49. Parolin M, Simonelli A, Cristofalo P, Sacco M, Bacciardi S, Maremmani AG, Cimino S, Trumello C, Cerniglia L. Drug addiction and emotional dysregulation in young adults. Heroin Addict Relat Clin Probl. 2017;19(3):37-48.

50. Craig L et al. Is emotional intelligence impaired in ecstasy-polydrug users? J Psychopharmacol. 2010;24(2):221-31. https://doi.org/10.1177/0269881108095713

51. Fox H. C, Axelrod SR, Paliwal P, Sleeper J, Sinha R. Difficulties in emotion regulation and impulse control during cocaine abstinence. Drug Alcohol Depend. 2007;89(2-3):298-301. https://doi.org/10.1016/j.drugalcdep.2006 .12 .026

52. Sánchez-Teruel D, Robles-Bello MA. Instrumentos de evaluación en inteligencia emocional: una revisión sistemática cuantitativa. Perspectiva Educacional. 2018;57(2):27-50. https://doi.org/10.4151/07189729

53. Resurrección DM, Salguero JM, Ruiz-Aranda D. Emotional intelligence and psychological maladjustment in adolescence: a systematic review. J Adolesc. 2014;37(4):461-72. https://doi.org/10.1016/j.adolescence.2014.0 3.012

\section{Notas}

* Artículo de revisión integrativa

Conflicto de intereses: los autores declaran que no existen conflictos de intereses.

Financiamiento: ninguno

\section{Licencia Creative Commons CC BY 4.0}

Cómo citar este artículo: Villarreal Mata JL, Navarro Oliva EIP, Muñoz Maldonado GE, Rodríguez Puente LA, Pacheco Pérez LA. Habilidades de la inteligencia emocional en relación con el consumo de drogas ilícitas en adultos: una revisión sistemática. Investig Enferm Imagen Desarr. 2020;22. https://doi.org/10.11144/Ja veriana.ie22.hier 